\title{
Calidad de Vida en Conductores de Taxis Colectivos Usando el Cuestionario Short Form 36 Versión 2
}

\section{QUALITY OF LIFE IN TAXIS DRIVERS USING THE QUESTIONNAIRE SHORT FORM 36 VERSION 2}

\author{
Eduardo Cerda Díaz', Héctor Hernán Rodríguez Bustos², Felipe David Leveke González³, Sebastián Israel Reyes Martínez² \\ Giovanni Olivares Péndola ${ }^{4}$ \\ 1. Laboratorio de Ergonomía, Escuela de Kinesiología, Facultad de Medicina Universidad de Chile. \\ 2. DMV. Msc. DBM-PhD, Diplomado Universidad de Chile Santiago, Chile \\ 3. Departamento de Kinesiología, Facultad de Medicina, Universidad de Chile, Santiago, Chile. \\ 4. Klgo, Laboratorio de Ergonomía, Departamento de Kinesiología, Facultad de Medicina, Universidad de Chile, Santiago, Chile.
}

\section{RESUMEN}

El presente estudio -no experimental, transversal y descriptivotiene por objetivo determinar la calidad de vida mediante el cuestionario de salud SF-36v.2 en conductores de taxis colectivos en Santiago de Chile. La recolección de datos fue realizado entre septiembre y noviembre del año 2013 a 76 conductores de la Empresa de Transportes Unidos de Maipú (ETRUM) S.A, seleccionados mediante participación voluntaria. La técnica de muestreo es mediante registro de información y el muestreo fue no aleatorio. Se utilizó el cuestionario autoadministrado SF-36v.2, para evaluar el perfil de estado de salud respecto a su calidad de vida, calculándose puntuaciones netas que se estandarizaron con la población chilena. La edad media de los participantes fue de 48,1 años $(\mathrm{DE}=10,95)(\mathrm{DE}=$ Desviación Estándar). Las dimensiones más afectadas con valores crudos fueron Salud General, Vitalidad, Dolor Corporal y Función Social. Al estandarizar los datos con la población chilena, las dimensiones más afectadas fueron Salud General, Vitalidad, Función Física y Función Social. Sin embargo, las diferencias encontradas al estandarizar los datos no superan el 0,5 de DE respecto a la media de referencia, lo que no supone significancia clínica.

(Cerda E, Rodríguez H, Leveke F, Reyes S, 2015. Calidad de Vida en Conductores de Taxis Colectivos Usando el Cuestionario Short Form 36 Versión 2. Cienc Trab. Ene-Abr; 17 [52]: 43-48).

Palabras clave: CALIDAD DE VIDA, CONDUCCIÓN DE AUTOMÓVIL, ESTADO DE SALUD.

\section{ABSTRACT}

This is a non experimental transversal, descriptive. Our aim is to determine the quality of life using the health questionnaire SF-36v.2 in taxi drivers in Santiago de Chile. Data collection was conducted between September and November 2013. -76 drivers from the United Transport Company of Maipú (ETRUM) S.A., were selected through voluntary participation. The sampling technique is a non random registration of the information. The self-administered questionnaire SF-36v.2 was used to assess the health status profile regarding their quality of life, the overall scores were calculated and standardized with the Chilean population. The average age was 48,1 years (SD = $10,95)(\mathrm{SD}=$ Standard Deviation).

The most affected dimensions on raw values are "General Health", "Vitality", "Body Pain" and "Social Function". Once standardized, the most affected dimensions were "General Health", "Vitality", "Physical Function" and "Social Function". However, the differences found by standardizing the data do not exceed 0,5 SD from the mean baseline, which means a lack of clinical significance.

Key words: QUALITY OF LIFE, AUTOMOBILE DRIVING , HEALTH STATUS .

\section{INTRODUCCIÓN}

La determinación de la calidad de vida o del estado de salud de las personas, en función de la definición de salud de la Organización Mundial de la Salud, es un fenómeno que cada día adquiere mayor importancia en la literatura médica. La calidad de vida se define como la "percepción que tienen los individuos acerca de su posi-

Correspondencia / Correspondence:

Eduardo Cerda Díaz

Dirección postal: 8380453

Tel.: (56 2) 29786513 / (56 2) 29786515 • Fax: (56 2) 29786514

e-mail: encerda@med.uchile.cl

Recibido: 12 de Julio de 2014 / Aceptado: 03 de Febrero de 2015 ción en la vida, teniendo en cuenta el contexto del sistema cultural y de valores en el que viven y en relación con sus metas, expectativas, normas e intereses". ${ }^{1}$ Ahora bien, la característica principal de la calidad de vida es la gran variedad de definiciones que presenta, puesto que integra variados elementos que no permiten que se obtenga una definición específica. Junto con lo anterior, la esencia de este concepto está en reconocer que la percepción de las personas sobre su estado de bienestar físico, psíquico, social y espiritual depende en gran parte de sus propios valores y creencias, su contexto cultural e historia personal. ${ }^{2}$

La incorporación de medidas de la percepción del estado de salud de los individuos en actividades de la vida cotidiana es objeto de creciente interés por parte de investigadores de politicas y gestión en el sistema de salud. ${ }^{3}$ La investigación sobre percepción de calidad de vida en personas con problemas de salud se torna altamente relevante, ya que puede constituirse como una categoría organizadora y de referencia al momento de establecer medidas que permitan 
generar cambios en los sistemas de trabajo. Este hecho demuestra que se hace necesario contar con instrumentos que evalúen la calidad de vida, especialmente validados para este tipo de poblaciones. ${ }^{4}$ En tanto, según el cuestionario de evaluación de riesgos psicosociales en el trabajo SUSESO - ISTAS 21 la relevancia para la salud de todas y cada una de las dimensiones psicosociales entre las diferentes ocupaciones y sectores de actividad puede ser distinta, pero el uso de las mismas definiciones e instrumento de medida posibilita las comparaciones entre todas ellas. ${ }^{5}$

La falta de un sistema común de información en Chile que contenga datos sobre enfermedades musculoesqueléticas (incluyendo su diagnóstico, factores de riesgo presentes en los puestos de trabajo y actividad, tiempos de exposición al factor de riesgo relacionado con la patología, actividad económica donde se presenta la exposición), contribuye a que la identificación, evaluación, control y seguimiento sea una tarea difícil. Entre los cuestionarios de salud que se utilizan para evaluar la salud de los trabajadores se encuentra el SF-36 v.2, que sirve para determinar el estado de salud de la persona examinada. ${ }^{6}$ El diseño del SF-36 v.2 incluye preguntas capaces de evaluar un espectro del estado de salud en un rango de estados desde "menos sanos" a "más sanos" de los encuestados. Actualmente, por su utilidad y simpleza, el cuestionario SF-36 es el más usado en estudios de estado de salud a nivel mundial. ${ }^{3}$ Además, el SF-36 es generalmente considerado en las investigaciones de EE.UU. como el criterio estándar de medidas genéricas de calidad de vida y se utiliza frecuentemente en la investigación y la práctica de la rehabilitación. ${ }^{7}$

De acuerdo con Karazek, Gardell \& Lindell, el estrés laboral y los problemas de salud están relacionados con la carga de trabajo, claridad frente a las tareas que se deben realizar, y los conflictos laborales, pero el efecto de estas características se ven moderadas por el control, oportunidades de desarrollo profesional y el apoyo social en el trabajo. ${ }^{8}$ La combinación de alta demanda y bajo control en el trabajo supone que es estresante, relacionándose con efectos adversos para la salud. Por otra parte, la organización del proceso de trabajo influye en los patrones de carga física, en las características psicosociales, como las demandas laborales y en la utilización de habilidades. ${ }^{9}$ Por ejemplo, la incapacidad de modificar o variar el ritmo de trabajo, o cambiar la frecuencia de la tarea cuando se producen molestias musculoesqueléticas genera estrés psicológico que repercute negativamente en la homeostasis fisiológica. Frente a lo anterior, los conductores por ser personas laboralmente activas están sometidos al concepto de carga física definida como "el conjunto de requerimientos físicos a los que se encuentra sometido el trabajador durante su jornada laboral, dependiendo de las características de la tarea [...]" (por Hernández, Fernández \& Baptista), junto a cargas derivadas del ambiente y del puesto de trabajo. ${ }^{10}$ Dentro de las causas físicas que se describen en la literatura, varios agentes de estrés laboral han sido identificados como factores que contribuyen a la aparición y desarrollo de problemas musculoesqueléticos, pero los dos principales son estrés postural y exposición prolongada a la vibración de cuerpo entero. ${ }^{11}$ La evidencia epidemiológica demuestra que la exposición a estos factores se asocia con mayor riesgo de presentar dolor lumbar en relación a la población general. ${ }^{12-16}$

En relación al desempeño de los conductores durante la conducción del automóvil, las molestias más comunes que se mencionan tienen relación con el vehículo, las condiciones del camino y los pasajeros. Estos últimos a menudo presionan con preguntas, solicitudes y demandas durante el viaje, provocando agotamiento laboral. ${ }^{17}$ En otros países la vulnerabilidad de la salud de los conductores y su importancia para el rendimiento, han impulsado a gobiernos y empresas a proporcionar varios programas de salud, que por lo general involucran exámenes de salud regulares. ${ }^{18}$

La organización de trabajo en la línea de taxis colectivos 6067 de Santiago presenta una estructura constituida esencialmente por los conductores, cuya misión es transportar pasajeros de forma eficaz en el menor tiempo posible. El horario de trabajo en promedio es de 12 horas con pausas entre cada recorrido y la distancia recorrida es de $15 \mathrm{Km}$ aproximadamente, comenzando desde Metro La moneda (Comuna de Santiago) hasta Plaza de Maipú (Comuna de Maipú). Dentro del sistema de trabajo del conductor existen dos subsistemas: El subsistema de espera, en el cual el conductor debe aguardar hasta completar la capacidad máxima de pasajeros para emprender el viaje. Y el subsistema de transporte, en el que se traslada por una ruta determinada para llegar al destino que le mencione previamente el pasajero o el encargado de rutas de la línea. Además, en este subsistema se realiza el pago por el servicio entregado. ${ }^{19}$

Actualmente, se desconoce el estado de salud de conductores de locomoción colectiva en Chile, por lo cual se aborda una población de conductores de taxis colectivos de Santiago, presentándose como uno de los primeros estudios que abarca esta dimensión en el rubro. El propósito es analizar el comportamiento de las distintas dimensiones del estado de salud a través de la percepción de calidad de vida dentro de una población de conductores de taxis colectivos de Santiago, siendo el objetivo general determinar la calidad de vida mediante el cuestionario SF-36 v.2 en conductores de taxis colectivos.

\section{MÉTODO}

El presente estudio es de tipo no experimental, transversal, descriptivo. La técnica de muestreo es de tipo censo y el muestreo fue no aleatorio. Como población de estudio se escogió a los conductores de taxis colectivos de la Línea 6067 de Santiago de la Empresa de Transportes Unidos de Maipú (ETRUM) S.A., la cual trabaja bajo un orden establecido, respecto a los recorridos que deben realizar, los horarios de trabajo durante la jornada laboral y la tarifa que deben cobrar, factores que varían según la demanda de pasajeros.

Para formar parte de este estudio, los participantes deberán reunir las siguientes características:

- Ser conductores activos de la Línea 6067 de taxis colectivos.

- Presentar mínimo 1 año en el puesto de trabajo.

- Género masculino.

- Consentimiento firmado.

Los criterios de exclusión son los siguientes:

- Se presente en un estado en el cual no se comprendan las preguntas del cuestionario (a causa de tratamiento farmacológico, estado de intemperancia).

- Presencia de banderas rojas (con síntomas constitucionales como pérdida de peso sin causa aparente, fiebre, escalofríos).

\section{Mediciones}

La recolección de datos del perfil del estado de salud de los conductores realizada entre septiembre y noviembre del año 2013 consiste en la aplicación de un cuestionario de calidad de vida. Las encuestas se llevan a cabo de forma voluntaria durante los tiempos de espera de pasajeros ("subsistema de espera"); de este modo se 
evita interferir con el ciclo de trabajo establecido. Junto con solicitar la colaboración de los conductores, a cada uno se le entrega el consentimiento informado, en el cual ellos entienden a cabalidad cuál es su rol en el estudio, concordando a la vez con sus principios y valores teniendo presente los riesgos inherentes y los beneficios que conlleva ser parte de este proyecto. Todo esto se hace siempre bajo la supervisión del mismo evaluador, el cual informa a los conductores sobre el proyecto de investigación y les explica en qué consiste su participación.

Luego de la aprobación y firma del consentimiento informado por parte del conductor, se le entrega el cuestionario de calidad de vida SF-36 v.2, que debe ser contestado de forma autónoma y sin intervención de terceros, siendo de su responsabilidad la comprensión de las preguntas y elección de las alternativas de respuesta. ${ }^{20}$

\section{Dimensiones SF-36 v.2}

La variable calidad de vida se mide a través del cuestionario SF-36 v.2, diseñado por el Medical Outcomes Study para medir percepción de calidad de vida, el cual ha sido validado en diferentes grupos poblacionales cumpliendo con criterios de relevancia y validez. El test detecta tanto estados positivos como negativos de salud. El contenido de los ítems se centra en el estado funcional y el bienestar emocional. Además, el ámbito de aplicación abarca estudios descriptivos y de evaluación. Consta de 36 items, que exploran 8 dimensiones del estado de salud: función física (FF), función social (FS), rol físico (RF), rol emocional (RE), salud mental (SM), vitalidad (VT), dolor corporal (DC) y percepción de la salud general (SG). Existe una pregunta, no incluida en estas ocho categorias, que explora los cambios experimentados en el estado de salud en el último año. ${ }^{4}$

El cuestionario no ha sido diseñado para generar un índice global, pero permite el cálculo de dos puntuaciones resumen mediante la combinación de las puntuaciones de cada dimensión: componente de salud físico (CSF) y componente de salud mental (CSM). Las propiedades psicométricas del cuestionario SF-36 v.2 han sido ampliamente estudiadas y han demostrado ser fiables, válidas y sensibles, tanto en su versión original como en la española, y tanto en población general como en pacientes con diferentes afecciones. ${ }^{21}$

\section{Análisis Estadístico}

El análisis estadístico de este estudio se realizó usando solamente aquellas encuestas que contenían el 100\% de respuestas contestadas, y fueron tabuladas y analizadas con el software Microsoft Office Excel 2007. Para el cálculo de las puntuaciones, los ítems de cada una de las 8 dimensiones se codifican, agregan y transforman en una escala de 0 (el peor estado de salud para esa dimensión) a 100 (el mejor estado de salud), o puntuación cruda. Para su interpretación, los valores se comparan con las normas poblacionales de Chile, lo que permite identificar desviaciones en los valores obtenidos. ${ }^{3}$

En primer lugar, se calculó la puntuación cruda para cada una de las ocho dimensiones de la población estudiada, la que luego se comparó con la puntuación cruda de la población general de referencia.

En segundo lugar, para evaluar si existe significancia clínica entre la población estudiada y la población general, se obtuvieron las puntuaciones estandarizadas con los valores de las normas poblacionales (norm-based scores), para las ocho dimensiones y los dos componentes sumarios del SF-36 v.2. La estandarización de las puntuaciones se realizó calculando la diferencia entre la puntuación cruda de cada conductor y la puntuación media poblacional, dividida por la DE poblacional correspondiente (puntuaciones z). Las puntuaciones $\mathrm{z}$ así obtenidas indican las unidades de DE que la puntuación de calidad de vida de una persona se aleja de la media poblacional. Estas puntuaciones z luego fueron reescaladas a una distribución con media de 50 y desviación típica de 10, para obtener las puntuaciones estandarizadas. ${ }^{20 ; 21}$

Si la diferencia entre la población estandarizada de una persona y la media poblacional supera el valor de 5 puntos (puntuación $\mathrm{z}>$ $0,5 \mathrm{DE}$ ), esta diferencia, mínimamente importante, significa que hay un cambio clínico real percibido por la persona más allá de la significancia estadistica. $^{22}$

\section{Aspectos éticos}

Este estudio cuenta con la aprobación del Comité de Ética de investigación en seres humanos de la Facultad de Medicina de la Universidad de Chile.

\section{RESULTADOS}

\section{Características generales de la muestra}

En este estudio la población a estudiar estuvo conformada por 121 personas, de las cuales 88 accedieron a contestar la encuesta. De las 88 encuestas, 12 no fueron correctamente contestadas, ya sea por preguntas omitidas o por elección de dos o más alternativas, de modo que el análisis del estudio se hizo con una muestra de 76 conductores, quienes respondieron en su totalidad el cuestionario SF-36 v.2, equivalente al 62,8\% de la población del total. El promedio de edad de la muestra fue de 48,1 años, con una desviación estándar (DE) igual a 10,95; con un rango de 28 a 69 años. La Tabla 1 muestra la distribución etaria de la muestra.

Los resultados estadísticos de todas las escalas son presentados en la Tabla 2. La media de las escalas de SF-36 v.2 se encuentran entre los valores 58,8 (SG) y 84 (FF), mientras que la mediana entre 60 (SG) y 87,5 (RF).

\section{Tabla 1.}

Distribución etaria de conductores.

\begin{tabular}{lcc|} 
RANGO & $N^{\circ}$ Total & $\%$ \\
$<40$ & 21 & 27,6 \\
$41-49$ & 23 & 30,3 \\
$50-59$ & 16 & 21,05 \\
$>60$ & 16 & 21,05 \\
Total & 76 & 100 \\
\hline
\end{tabular}

Tabla 2. Estadísticas descriptivas de las escalas.

\begin{tabular}{|c|c|c|c|c|c|c|c|c|}
\hline & $\% \mathrm{FF}$ & $\% \mathrm{RF}$ & $\% D C$ & $\%$ SG & $\% \mathrm{VT}$ & $\% \mathrm{FS}$ & $\%$ RE & $\%$ SM \\
\hline Media & 84 & 81,2 & 67,9 & 58,8 & 59,4 & 71,2 & 76,9 & 70,9 \\
\hline IC $(95 \%)$ & $80,5-87,5$ & $77,0-85,3$ & $62,0-73,8$ & $54,2-63,4$ & $54,4-64,5$ & $66,0-76,5$ & $72,4-81,3$ & $66,0-75,7$ \\
\hline Mediana (percentil 50) & 85 & 87,5 & 68,75 & 60 & 62,5 & 75 & 75 & 70 \\
\hline Desviación Estándar (DE) & 15,14 & 18,07 & 25,71 & 20,11 & 22,09 & 22,91 & 19,51 & 21,17 \\
\hline
\end{tabular}

$\% \mathrm{FF}=$ Porcentaje Función Física. \%RF = Porcentaje Rol Físico. \%DC = Porcentaje Dolor Corporal. \%SD = Porcentaje Salud General. \%VT = Porcentaje Vitalidad

$\%$ FS = Porcentaje Función Social. \%RE = Porcentaje Rol Emocional. \%SM = Porcentaje Salud Mental. IC = Intervalo de Confianza del 95\%. 


\section{Gráfico 1.}

Valores crudos de las medias de las 8 dimensiones de salud en conductores y población chilena.

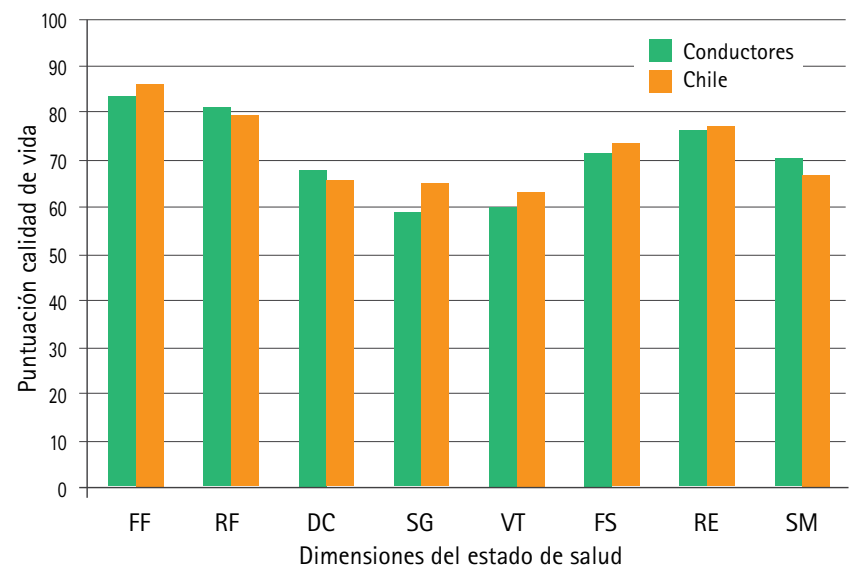

FF $=$ Función Física, RF $=$ Rol Físico, $\mathrm{DC}=$ Dolor Corporal, $\mathrm{SD}=$ Salud General $\mathrm{VT}=$ Vitalidad, $\mathrm{FS}=$ unción Social, $\mathrm{RE}=$ Rol Emocional, $\mathrm{SM}=$ Salud Mental.

\section{Gráfico 2.}

Valores crudos de las medias de los CSF y CSM en conductores y población chilena.

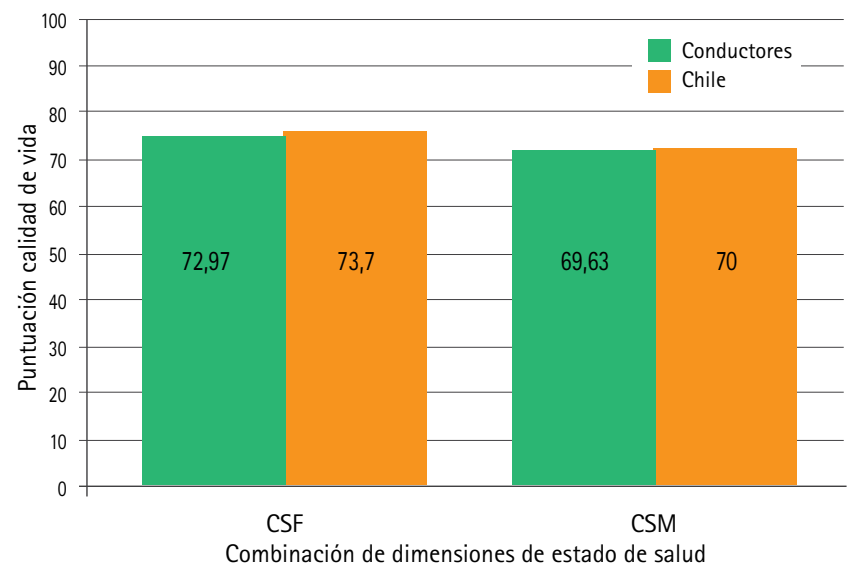

CSF = Componente de Salud Físico, CSM = Componente de Salud Mental.

\section{Gráfico 3.}

Valores crudos de las medias de los CSF y CSM en conductores categorizados por edad.

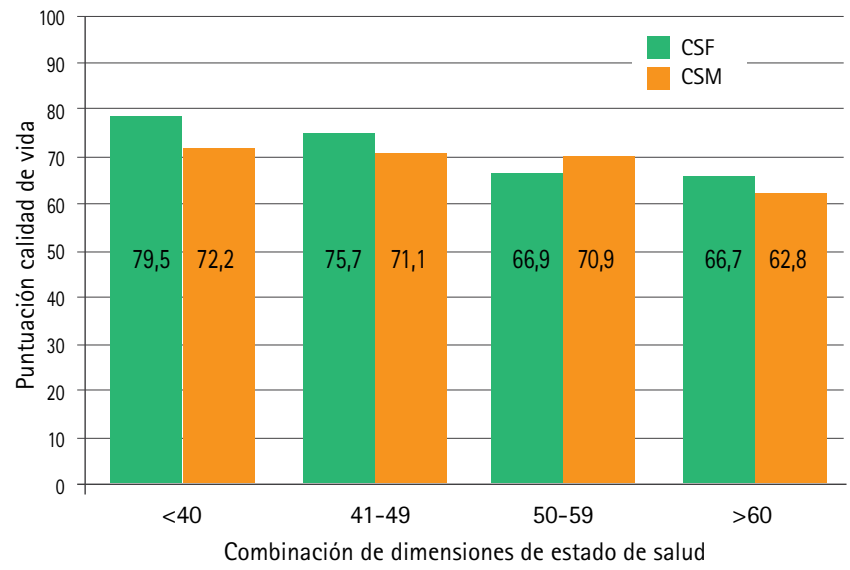

$\mathrm{CSF}=$ Componente de Salud Físico, CSM = Componente de Salud Mental

\section{Puntuaciones de la calidad de vida}

El Gráfico 1 muestra la media de los valores crudos (ponderados de 0 - 100) de la muestra obtenida de los conductores de taxis colectivos y los de referencia de Chile ${ }^{3}$ en las 8 dimensiones de salud, evaluada por el SF-36 v.2, donde 0 representa el peor estado de salud posible y 100 el mejor estado de salud.

El Gráfico 2 muestra las 8 dimensiones agrupadas en CSF y CSM para los conductores y la población de referencia. En el CSF, el valor para los conductores es de 73,0, mientras que el valor de referencia de Chile es 73,7; y en el CSM, los valores son 69,6 y 70, respectivamente. Y en el Gráfico 3, se aprecian los valores crudos de las medias categorizados por edad en la población de conductores.

En los gráficos siguientes y para simplificar la interpretación de los resultados, se usan los resultados normalizados (estandarizados con la población de Chile 2004-2005) de las escalas y medidas de resumen según lo recomienda la metodología de evaluación del cuestionario SF-36 v.2. ${ }^{20}$ Con los resultados normalizados -cada escala tiene una media de 50 y una desviación estándar de 10 puntos- las diferencias entre los resultados de las escalas reflejan mejor las diferencias en el estado de salud. Cada vez que una escala o medida de resumen está por debajo de 50, el estado de salud estará bajo el promedio y cada punto es $1 / 10$ de una desviación estándar. Una diferencia $>0,5 \mathrm{DE}$ con los valores de referencia supone significancia clínica. ${ }^{22}$

\section{Gráfico 4.}

Valores normalizados y estandarizados con la población chilena de las medias de las 8 dimensiones en conductores.

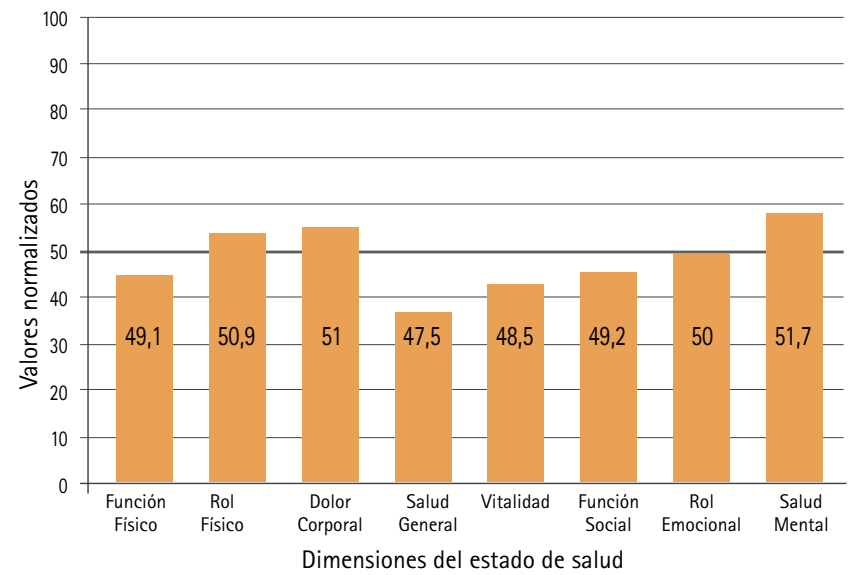

\section{Gráfico 5.}

Valores normalizados y estandarizados con la población chilena, de las medias de los componentes físico y mental de salud.

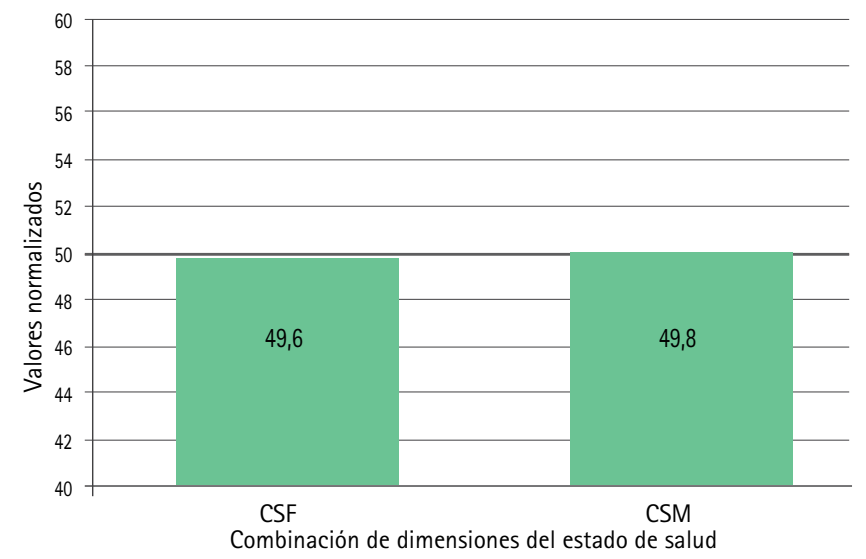


El Gráfico 4 muestra la distribución de los valores de las medias normalizadas para las 8 dimensiones de salud, mientras que el Gráfico 5 representa los valores normalizados de las medias de los CSF y CSM, 49,7 y 49,8, respectivamente.

\section{DISCUSIÓN}

Los estudios acerca de calidad de vida son numerosos en diferentes países; sin embargo, en Chile existen pocos estudios que aborden este tema con el fin de establecer valores epidemiológicos para la población en general. ${ }^{2,3,4,23,24,25}$

Los resultados de este estudio presentan, por primera vez, valores de calidad de vida relacionada con la salud en una población específica de conductores de taxis colectivos. El abarcar una población del rubro de transportes es relevante, ya que según diversos estudios se ha comprobado que existe una gran gama de factores a los que se encuentran expuestos, los cuales pueden ser desfavorables para su salud y percepción de calidad de vida. , $^{8-12-18}$

Se utiliza la media de cada una de las 8 dimensiones para la interpretación de los resultados, ya que en la gran mayoría de los estudios de calidad de vida (medidos con SF-36) son realizados de esta manera; por lo tanto, este es el método con el cual se puede llevar a cabo fácilmente comparaciones entre estudios.

La población estudiada se caracterizó por tener una mayor densidad de participantes entre las edades 41 y 49 años, con un 30,3\% de los sujetos; luego los menores de 40 años, con un 27,6\%; y posteriormente los sujetos entre 50 y 59 años con un 21,05\%, al igual que los mayores de 60 años, que presentaron un 21,05\% de la población total.

\section{Valores Crudos}

La población estudiada presenta una distribución de las medias de las 8 dimensiones similar a la observada para la población de referencia, en donde los valores crudos de estas, en orden ascendente, son: Salud General (SG), Vitalidad (VT), Dolor Corporal (DC), Función Social (FS), Salud Mental (SM), Rol Emocional (RE), Rol Físico (RF) y, por último, Función Física (FF). Los valores mínimo y máximo son 58,8\% y $84 \%$, respectivamente. En estas dimensiones, al ser agrupadas en CSF y CSM, se obtienen 73\% y 69,6\%, respectivamente. ${ }^{3}$

En los valores crudos, al ser analizados por segmentos etarios, se evidencia que los sujetos con mayor edad presentan peor calidad de vida. Una característica observada es que el componente mental es menor al físico en todos los segmentos, a excepción de los sujetos que se encuentran en el rango etario entre 50-59. La tendencia de disminución en la calidad de vida a medida que aumenta la edad es concordante con los valores obtenidos en la población de referencia utilizada, sin embargo, no se pudo establecer comparaciones numéricas, ya que el documento solo proporciona gráficos respecto a esto y no valores específicos.

\section{Valores normalizados y estandarizados a la población chilena}

Lo descrito anteriormente tiene relación con los valores crudos obtenidos; sin embargo, la recomendación entregada es interpretarlos una vez estos hayan sido estandarizados con la población de referencia. ${ }^{20}$ Con los datos disponibles, se pudo estandarizar los valores para las ocho dimensiones y para los componentes físico y mental (Gráficos 4 y 5), y se establece que los valores que se encuentran por debajo de 50 (ya estandarizados) presentan peor calidad de vida que la media de la población de referencia.
Según esta interpretación, los 5 valores que están por debajo de la media de referencia, en orden ascendente, son: SG, VT, FF, FS y RE. Mientras que los valores que se encuentran por sobre la media de referencia, en orden ascendente, son: RF, DC y SM.

Si se comparan estos resultados, una vez estandarizados, con los valores crudos se observa que lo que se puede considerar como ámbito afectado variará. Por ejemplo, SG en ambas interpretaciones se encuentra como el valor más bajo, lo que reafirma que es el aspecto más afectado. Luego, lo mismo ocurre con VT, que en ambas interpretaciones es el segundo más afectado. Sin embargo, a partir del tercero hay diferencias en ambas formas de interpretarlo: si bien en los valores crudos el siguiente valor más afectado es DC al compararlo con la población de referencia, esto no debería ser un factor preocupante, ya que estaría por sobre la media de la población, inclusive sería el segundo valor más alto de los ocho. Algo similar ocurre con FF, pero a la inversa, ya que a pesar que es el valor crudo más alto de los ocho, al momento de normalizar este es el tercer valor más bajo, o sea, se encuentra bajo la media de la población. Lo mismo ocurre con los otros ámbitos, pero es menos notorio.

Sin embargo, a pesar de lo expuesto anteriormente, existe significancia clínica cuando estos valores están a 0,5 DE de la media (22). En este caso, 0,5 DE son equivalentes a 5 puntos, por lo tanto, los valores que se encuentren por debajo de 45 serán considerados como clínicamente significativos. Si se aprecian los valores obtenidos por cada uno de los 8 ámbitos, inclusive por los componentes mental y físico, se puede notar que no existen valores que varien más de 0,5 DE de la media.

En conclusión, los valores obtenidos en la población de conductores presentan tendencias similares a la población chilena a la hora de comparar valores crudos, tanto en distribución etaria relacionada a calidad de vida, como distribución de la media de cada ámbito. Además, si se estandarizan los valores con la población chilena pueden apreciarse los valores que se encuentran por debajo de la media, y que al compararlo con los valores crudos el orden de alteración de algunos ámbitos es distinto. Sin embargo, las diferencias existentes entre la población de conductores y la población de referencia no logran ser significativas clínicamente.

\section{Limitaciones del estudio}

Las principales limitaciones tienen que ver fundamentalmente con 3 aspectos. En primer lugar, la cantidad de variables incluidas en este estudio eran acotadas, siendo estas la edad y la calidad de vida. Sin embargo, se podría haber recolectado datos acerca de otros aspectos, como: nivel educacional, previsión de salud, horas trabajadas (a pesar de ser variable), entre otros.

En segundo lugar, el tamaño de la muestra, al ser un grupo acotado de la realidad nacional, no es suficiente para extrapolar los resultados a otros grupos de conductores en este y otros rubros, lo que limita las acciones que se puedan tomar respecto a los resultados. Además, como no se pudo encuestar al 100\% de la población (solo al 72,7\% del total), junto con el hecho de que no se contestaron correctamente todas las encuestas, solo fue posible obtener como datos útiles el $62,8 \%$ de la población total, lo que puede suponer que hubo sesgo de no respuesta o efecto del voluntario, en el que se corre el riesgo de que se automarginen los participantes, por ejemplo, para no ser sancionados socialmente o bien, pueden excluirse los sujetos que se sienten sanos y no desean ser evaluados.

En último lugar, el no contar con acceso libre a la base de datos nacional en forma pública, no permite realizar mayores comparaciones entre las variables analizadas, ya que en el estudio de refe- 
rencia no se exponen explícitamente todos los valores obtenidos para las variables, por ejemplo los obtenidos por rango etario, ya que solo se encuentran los gráficos, limitando de esta manera posibles comparaciones y análisis respecto al tema.

\section{Proyecciones}

El presente proyecto intenta aportar información relevante del perfil de salud de los conductores de taxis colectivos para describir los aspectos relevantes de la calidad de vida. Es relevante tener presente el nivel de calidad de vida en futuros estudios de poblaciones laboralmente activas que presenten factores de riesgos tanto físicos como psicológicos, a fin de realizar intervenciones que permitan hacer modificaciones en el sistema o en el entorno de trabajo para evitar, por ejemplo, el desarrollo de trastornos musculoesqueléticos.

Esta investigación constituye un primer paso de investigación en el área, a lo menos en Chile, permitiendo que en futuras investigaciones se pueda incluir una mayor cantidad de variables que se puedan identificar en la población de estudio, con el objeto de obtener información que permita comparar, y además extrapolar, a poblaciones mayores. Por ejemplo, aplicar el cuestionario de calidad de vida SF-36 v.2 en conductores de otros medios como buses del transporte público o privado. Además, sería interesante determinar la relación entre el nivel de calidad de vida y presencia de dolor lumbar inespecífico, junto con la incapacidad física consecuente del dolor lumbar, ya que existe vasta evidencia que respalda la alta prevalencia de esta condición en los conductores de algún medio de transporte. ${ }^{13 ; 15 ; 16}$

\section{REFERENCIAS}

1. The WHOQOL group. The World Health Organization Quality of Life assessment (WHOQOL).Position Paper from the World Health Organization.SocSci Med [en linea].1995 [citado 28 mar 2014]; 41(10):1403-1409. Disponible en: http://www. sciencedirect.com/science/article/pii/027795369500112K?_rdoc=1\&_fmt=high\&_ origin=ihub\&_docanchor=\&md5=9ffa87934275edd7180b52f5e973f002.

2. Schawartzmann L. Calidad de vida relacionada con la salud: Aspectos conceptuales. Ciencenferm [en línea]. 2003 [citado 28 mar 2014];9(2):9-21. Disponible en: http://www.scielo.cl/pdf/cienf/v9n2/art02.pdf

3. Superintendencia de Isapres. Estado de salud de beneficiarios del sistema de salud de Chile: 2004-2005 [en línea]. 7 de agosto de 2006 [citado 28 mar 2014]. Disponible en: http://www.supersalud.gob.cl/documentacion/569/articles-1062 _recurso_1.pdf

4. Dois A, Contreras A. Validación de una escala de calidad de vida en un grupo de personas con esquizofrenia de la región metropolitana-Chile. Ciencenferm [en línea].2007 [citado 28 mar 2014]; 13(1):35-44. Disponible en: http://www. scielo.cl/scielo.php?pid=S0717-95532007000100005\&script=sci_arttext .

5. Chile. Ministerio del Trabajo y Seguridad Social. Superintendencia de Seguridad Social. SUSESO-ISTAS 21 Cuestionario de Evaluación de Riesgos Psicosociales en el Trabajo [en línea]. Santiago: Ministerio del Trabajo y Seguridad Social; 2013[citado 28 mar 2014].Disponible en: http://www.achs.cl/portal/Empresas/ Documents/MANUAL\%20SUSESO\%20ISTAS\%2021\%20Versi\%C3\%B3n\%20 Breve.pdf\#_blank

6. Chile. Ministerio de Salud.División de políticas saludables y promoción.Protocolo de vigilancia para trabajadores expuestos a factores de riesgo de trastornos musculoesqueléticos de extremidades superiores relacionados con el trabajo [en línea]. Santiago: MINSAL;2012 [citado mar 2014].Disponible en: http://web. minsal.cl/portal/url/item/dbd6275dd3c8a29de040010164011886.pdf

7. Horner-Jhonson W, Krahn G, Suzuki R. Differential Performance of SF-36 Items in Healthy Adults With and Without Functional Limitations. ArchPhysMedRehabil [on line]. 2010[cited2014 Mar 28];91(4):570-575. Availablefrom: http://www. sciencedirect.com/science/article/pii/S0003999310000092?via=ihub.

8. Karazek $R$, Gardell $B$, Lindell J. Work and non-work correlates of illness and behaviour in male and female Swedish white collar workers. J OccupBehav [on line]. 1987[cited2014 Mar 28];8(3):187-207. Disponible en internet: http:// onlinelibrary.wiley.com/doi/10.1002/job.4030080302/abstract?deniedAccessCus tomisedMessage $=\&$ userlsAuthenticated $=$ false.

9. Punnett L, Wegman D. Work-related musculoskeletal disorders: the epidemiologic evidence.J ElectromyogrKinesiol[on line]. 2004 [cited 2014 Mar 28];14 (1):13-23. Availablefrom: http://www.journals.elsevierhealth.com/periodicals/ jek/article/PIIS1050641103001251/abstract.

10. Hernández $R$, Fernández $C$, Baptista $P$. Metodología de la investigación. $3^{\circ}$ ed. México DF: McGraw Hill; 2004.

11. Okunribido O, Shimbles S, Magnusson M. City bus driving and low back pain: A study of the exposures to posture demands, manual materials handling and whole-body vibration. ApplErgon [on line]. 2007 [cited 2014 Mar 28]; 38(1):29-38. Availablefrom: http://www.sciencedirect.com/science/article/pii/S000368 7006000172.

12.-Magnusson $M$, Pope $M$, Wilder $D$, Areskoug B. Are occupational drivers at an increased risk for developing muskuloskeletal disorders? Spine[on line].1996 [cited 2014 Mar 28];21(6): 710-717. Availablefrom: http://www.ncbi.nlm.nih. gov/pubmed/8882693.

13. Pope M, Lim K, Magnusson M. Spine Ergonomics. Annu Rev Biomed Eng. 2002; 4:49-68

14. Pérez J. Contribución al estudio de la lumbalgia inespecífica. Rev Cubana OrtopTraumatol [en línea]. 2006 [citado 2014-03-28]; 20(2):00. Disponible en: http://scielo.sld.cu/pdf/ort/v20n2/ort10206.pdf

15. Lis A, Black K. Association between sitting and occupational LBP. EurSpine J [on line].2007 [cited 2014 Mar 28]; 6:283-298. Availableon: http://www. ncbi.nlm.nih.gov/pmc/articles/PMC2200681/pdf/586_2006_Article_143.pdf

16. Roffey $D$, Wai $E$, Bishop P. Causal assessment of awkward occupational postures and low back pain: results of a systematic review. Spine J [on line]. 2010 [cited 2014 Mar 28];10(1): 89-99. Available from: http://www.ncbi.nlm. nih.gov/pubmed/19910263.

17. Chen $\mathrm{C}, \mathrm{Kao} Y$. The connection between the hassles-burnout relationship, as moderated by coping, and aberrant behaviors and health problems among bus drivers.Accid Anal Prev.2013; 53:105-111.

18. Chung Y, Wong J. Developing effective professional bus driver health programs: An investigation of self-rated health. Accid Anal Prev.2011; 43(6):2093-2103..

19. Zúñiga M, Molina K. Relaciones laborales de los choferes del Transantiago [en línea]. Santiago: ICAL; 2013[citado 28 mar 2014]. (El trabajo hoy). Disponible en:http://www.ical.cl/wp-content/uploads/Transantiago1.pdf

20. Ware J. SF-36 Health Survey Update. En: Maruish M, editor.The Use of psychological testing for treatment planning and outcomes assessment. New Jersey: Lawrence Erlbaum Associates; 2004. pp. 693-718.

21. Rodríguez-Romero B, Pita S, Pertega S. Calidad de vida relacionada con la salud en trabajadoras del sector pesquero usando el cuestionario SF-36. GacSanit [en linea]. 2013[citado 28 mar 2014]; 27(5):418-424. Disponible en: http://scielo. isciii.es/scielo.php?script=sci_arttext\&pid=S0213-91112013000500007\&Ing=e s\&nrm=iso\&tlng=es.

22. Norman G, Sloam J, Wyrwich K. Interpretation of changes in health-related quality of life: the remarkable university of half a standard deviation. MedCare [on line]. 2003 [cited 2014 Mar 28]; 41(5):582-92. Availablefrom: http://www. ncbi.nlm.nih.gov/pubmed/12719681. PMID: 12719681.

23. Cardona D, Agudelo H. Construcción cultural del concepto calidad de vida. Revista Facultad Nacional de Salud Pública [en línea]. 2005[citado 28 mar 2014]; 23(1):8090. Disponible en: $\mathrm{http}: / /$ www.redalyc.org/articulo.oa?id= 12023108.

24. Urzúa A, Caqueo-Urizar A. Calidad de vida: Una revisión teórica del concepto. Ter Psicol [en línea]. 2012[citado 28 mar 2014]; 30(1):61-71. Disponible en http://www.scielo.cl/scielo.php?pid=S0718-48082012000100006\&script=sci_ arttext.

25. Vilaguta G, Ferrera M, Rajmilb L. El Cuestionario de Salud SF-36 español: una década de experiencia y nuevos desarrollos. GacSanit [en línea]. 2005[citado 28 mar 2014];19(2):135-50. Disponible en: http://scielo.isciii.es/scielo.php?pid= S0213-91112005000200007\&script=sci_arttext. 